\title{
Réduction de doses de cyperméthrine- triazophos et lutte raisonnée en culture cotonnière au Bénin
}

\author{
Djihinto Cocou Angelo*1, Affokpon Antoine1, Dannon Elie ${ }^{2}$ et Aboua Charlemagne ${ }^{1}$ \\ ${ }^{1}$ Institut National des Recherches Agricoles du Bénin, 01BP 884 Cotonou, République du Bénin \\ ${ }^{2}$ International Institute of Tropical Agriculture (IITA), 08-B.P. 0932, Tri Postal Cotonou, Bénin \\ *Auteur correspondant : DJIHINTO Cocou Angelo, Institut National des Recherches Agricoles du Bénin, 01BP 884 \\ Cotonou, République du Bénin. djihinto@yahoo.com; Tel : 0022997983485
}

Original submitted in on $9^{\text {th }}$ December 2015. Published online at www.m.elewa.org on 29th February 2016 http://dx.doi.org/10.4314/jab.v98i1.3

\section{RESUME}

Objectifs : L'étude a pour objectif de réduire les doses de cyperméthrine-triazophos en culture cotonnière et d'apporter ou non les doses complémentaires en fonction du niveau d'infestation des ravageurs.

Méthodologie et Résultats : La réduction de doses de cyperméthrine-triazophos a été étudiée sur la station de Gobé dans le Centre Bénin dans un dispositif factoriel à deux facteurs en bloc aléatoire complet de 6 répétitions. En milieu réel, la lutte raisonnée basée sur l'application de demi-dose d'insecticides avec l'apport de la demi-dose complémentaire en fonction du seuil d'infestation des ravageurs a été comparée à la protection classique du cotonnier dans un dispositif de bloc aléatoire complet de 10 répétitions. Aucune différence significative n'a été observée entre les doses de cyperméthrine allant de $15 \mathrm{~g} / \mathrm{ha}$ à $35 \mathrm{~g} / \mathrm{ha}$ lors du dénombrement des ravageurs rencontrés. Par contre, la dose de $75 \mathrm{~g} / \mathrm{ha}$ de triazophos est significativement inférieure $(P<0,05)$ à la dose de $150 \mathrm{~g} /$ ha pour le contrôle des populations de ces ravageurs. A la récolte sur station, aucune différence significative n'a été observée entre les rendements obtenus pour les doses $20 \mathrm{~g} / \mathrm{ha}$, $30 \mathrm{~g} / \mathrm{ha}$ et $35 \mathrm{~g} / \mathrm{ha}$ de cyperméthrine. De même, aucune différence significative n'a été obtenue entre les rendements fournis par les doses $75 \mathrm{~g} / \mathrm{ha}$ et $150 \mathrm{~g} / \mathrm{ha}$ de triazophos. En milieu réel, la lutte raisonnée a permis d'obtenir un rendement de coton graine significativement supérieur $(P<0,01)$ à celui de la protection classique. Conclusion et Application des Résultats : La réduction de $35 \mathrm{~g} / \mathrm{ha}$ à $20 \mathrm{~g} / \mathrm{ha}$ de la dose de cyperméthrine est possible sans affecter significativement la production. L'application de demi-dose d'insecticides par les agriculteurs avec l'apport de la demi-dose complémentaire en fonction du seuil d'infestation des ravageurs en lutte raisonnée procure un rendement de coton graine significativement supérieur $(P<0,01)$ à celui de la protection classique.

Reduced doses of cypermethrin- triazophos and pest management in cotton production in Benin Objectives: The study aims to reduce cypermethrin-triazophos doses used in the cotton crop production and to add or not the complementary doses according to pests infestation level.

Methodology and Results: Cypermethrin-triazophos doses reduction was studied in the Gobé station in the Centre of Benin in a complete randomized block design with combination of two factors and six repetitions. In the real environment, rational protection based on half-dose application of insecticides with the spraying of complementary half-dose according to pest infestation level has been compared to conventional protection of cotton crop in a complete randomized block design with ten repetitions. No significant difference was observed 
between cypermethrin doses ranging from $15 \mathrm{~g} / \mathrm{ha}$ to $35 \mathrm{~g} / \mathrm{ha}$ during the counting of observed insects. On the other hand, triazophos dose of $75 \mathrm{~g} / \mathrm{ha}$ was significantly $(P<0.05)$ lower than dose of $150 \mathrm{~g} / \mathrm{ha}$ for insect populations control. At harvest time in the station, no significant difference was observed between yields of cotton seed obtained with cypermethrin doses ranging from $20 \mathrm{~g} / \mathrm{ha}$ to $35 \mathrm{~g} / \mathrm{ha}$. Likewise, no significant difference was observed between yields of cotton seed obtained with triazophos doses of $75 \mathrm{~g} / \mathrm{ha}$ and 150 $\mathrm{g} / \mathrm{ha}$. In the real environment, rational protection produced a cotton seed yield significantly $(P<0.01)$ higher than yield provided by conventional protection.

Conclusion and Application of Results : The reduction of $35 \mathrm{~g} / \mathrm{ha}$ to $20 \mathrm{~g} / \mathrm{ha}$ dose of cypermethrin is possible without significantly affecting production. The application of half-dose of insecticides by farmers with the provision of additional half dose depending on the level of infestation of pests in pest management provides a cotton seed yield significantly higher $(P<0.01)$ to that of the conventional protection.

\section{INTRODUCTION}

Le cotonnier est l'une des plantes les plus attaquées par les ravageurs et la culture cotonnière au Bénin fait l'objet de plusieurs traitements insecticides chaque année. Les insecticides sont distribués aux producteurs par la Société Nationale pour la Promotion Agricole (SONAPRA) en fonction des superficies cotonnières pouvant atteindre 400.000 ha chaque année avec une production annuelle du coton graine qui avoisine 400.000 tonnes ces dernières années. La cyperméthrine est la molécule insecticide de la famille des pyréthrinoïdes la plus utilisée contre les Lépidoptères du cotonnier au Bénin (Djihinto, 2004). Les ravageurs des feuilles du cotonnier sont contrôlés par les insecticides de la famille des organophosphorés dont le triazophos est l'une des molécules insecticides les plus efficaces et les plus utilisées au Bénin (Djihinto, 2004). Les problèmes environnementaux associés à l'utilisation des insecticides chimiques appliqués en culture cotonnière au Bénin sont essentiellement l'apparition de la résistance des insectes aux insecticides (Djihinto et al., 2009 et 2013) et les cas d'intoxication des agriculteurs liés à la manipulation des produits chimiques (Tovignan et al., 2001). Faute d'études spécifiques, la pollution environnementale liée à l'application des pesticides sur le coton n'a pas été quantifiée au Bénin de même que l'effet de l'insecticide sur les insectes auxiliaires non ciblés.

\section{MATERIELS ET METHODES}

Le matériel végétal : La variété H279-1 du cotonnier Gossypium hirsutum a été utilisée dans les essais qui ont été réalisés tant sur station qu'en milieu réel.
Un autre aspect de la protection chimique du cotonnier est le coût parfois élévé des insecticides appliqués. Face à ces problèmes, les traitements insecticides et les doses appliquées ont besoin d'être repensés. Pour réduire les problèmes environnementaux et le coût des traitements insecticides, les pays d'Afrique Centrale et de l'Ouest tels que la République Centre Africaine, le Cameroun, le Tchad, la Côte d'Ivoire, le Togo, le Mali, le Bénin, le Burkina Faso ont développé des stratégies de lutte raisonnée en culture cotonnière comme la lutte étagée ciblée, la lutte sur seuil, la lutte après observation individuelle des chenilles visant à réduire la dose d'insecticide épandu tout en garantissant un rendement satisfaisant de coton graine produit (Silvie et Sognigbe, 1993; Vodounnon, 1995 ; Nibouche et al., 2003; Sinzogan et al., 2004; Prudent et al., 2007 ; Silvie et al., 2011 et 2013 ; Kpadé et Mensah, 2013; Brévault et al., 2014). Dans la même logique, des études de réduction des doses d'insecticides ont été réalisées dans le domaine de la lutte contre le criquet pèlerin (Amadou et Sidi, 2013) et la préservation d'insecte auxiliaire non ciblé par les traitements chimiques (Colignon et al., 2003). C'est dans ce cadre que des expérimentations de réduction de doses d'insecticides et de lutte raisonnée en culture cotonnière ont été entreprises au Bénin.

\section{Insecticides :}

Cyperméthrine: La formulation CE (Concentré Emulsionnable) de cyperméthrine a été expérimentée. Le produit commercial à base de cyperméthrine testé 
est Cypercal 87,5 CE (cyperméthrine 87,5 g/l). Le produit Cypercal 87,5 CE a été fourni par la société Arysta Life Science.

Triazophos: La formulation CE (Concentré Emulsionnable) de triazophos a été expérimentée. Le produit commercial à base de triazophos testé est Hostathion $400 \mathrm{CE}$ (triazophos $400 \mathrm{~g} / \mathrm{l}$ ). Le produit Hostathion 400 CE appartient à la société Bayer.

Méthodes: Un essai de réduction de dose de cyperméthrine-triazophos a été installé sur station de recherche et des essais de lutte raisonnée basée sur l'application de demi-dose d'insecticides avec un apport de la demi-dose complémentaire en fonction des seuils d'infestation des ravageurs ont été installés en milieu paysan.

Essai de réduction de dose de cyperméthrinetriazophos sur station de recherche : Pour l'essai de réduction de dose de cyperméthrine-triazophos sur station de recherche, un dispositif factoriel à deux facteurs en bloc aléatoire complet, comportant 6 répétitions avec des parcelles élémentaires de 9 lignes de 11 mètres de longueur dont 8 lignes traitées a été installé. Les deux modalités du premier facteur sont les deux doses de triazophos testées : $75 \mathrm{~g} / \mathrm{ha}$ et $150 \mathrm{~g} / \mathrm{ha}$. Les six modalités du second facteur sont les six doses de cyperméthrine testées : $0 \mathrm{~g} / \mathrm{ha}, 10 \mathrm{~g} / \mathrm{ha}, 15 \mathrm{~g} / \mathrm{ha}, 20 \mathrm{~g} / \mathrm{ha}$, $30 \mathrm{~g} / \mathrm{ha}$ et $35 \mathrm{~g} / \mathrm{ha}$. Les combinaisons des deux facteurs sont appliquées lors des six applications des doses mises en comparaison. Les applications insecticides ont eu lieu à partir de 50 jours après la levée du cotonnier. Deux applications insecticides successives sont espacées de 14 jours. Sur chaque parcelle élémentaire, les observations suivantes ont été réalisées :

- Nombre de plants attaqués par Syllepte derogata, Aphis gossypii, Bemisia tabaci et Polyphagotarsonemus latus sur 20 plants observés une fois par semaine à partir de 49 jours après la levée du cotonnier,

- Nombre de feuilles attaquées par Aphis gossypii, Bemisia tabaci et Polyphagotarsonemus latus sur 100 feuilles observées une fois par semaine à partir de 49 jours après la levée du cotonnier,

- Nombre de chenilles de Helicoverpa armigera, Diparopsis watersi, Earias spp, Cryptophlebia leucotreta, Pectinophora gossypiella, Spodoptera littoralis sur 20 plants observés une fois par semaine à partir de 49 jours après la levée du cotonnier,

- Nombre de capsules vertes saines, trouées, piquées, pourries et nombre de chenilles rencontrées dans 100 capsules vertes observées une fois par semaine à partir de 84 jours après la levée du cotonnier,
- Nombre de capsules mûres saines, trouées, tachées, momifiées et proportion de coton jaune dans 100 capsules observées juste après chaque récolte de coton graine,

- Récolte du coton graine des deux lignes centrales.

Essais de lutte raisonnée en milieu paysan : En milieu paysan, quatre essais de lutte raisonnée ont été installés dans quatre communes (Dassa, N'Dali, Nikki, Banikoara) couvrant les zones cotonnières du Bénin. Un dispositif de bloc aléatoire complet, comportant 10 répétitions et des parcelles élémentaires de $1000 \mathrm{~m}^{2}$ entièrement traitées, a été installé dans chacune des quatre communes cotonnières. La protection classique a été comparée à la lutte raisonnée. La protection classique est réalisée avec l'application de la pleine dose d'insecticides. La lutte raisonnée est basée sur l'application de demi-dose d'insecticides avec l'apport de la demi-dose complémentaire au cas où les seuils d'infestation des ravageurs pouvant affecter la culture sont atteints. Six seuils d'infestation ont été définis : le seuil « puceron », le seuil « Syllepte derogata », le seuil « acarien », le seuil " $H$. armigera », le seuil " autres carpophages " et le seuil "cumul des carpophages». Les trois premiers seuils se réfèrent à des nombres de plants présentant des symptômes d'infestation des ravageurs et les trois derniers concernent le nombre de chenilles rencontrées sur 40 plants observés par parcelle élémentaire. Ces seuils sont fixés respectivement à $33,10,3,5,10$ et 10 (Vodounnon, 1995 ; Prudent et al., 2007, Silvie et al., 2011 et 2013). Six applications insecticides ont été réalisées à partir de 50 jours après la levée du cotonnier pour la protection classique et la lutte raisonnée. Deux applications insecticides successives sont espacées de 14 jours. Une observation hebdomadaire permet de déterminer les seuils d'infestation des ravageurs afin d'appliquer ou non la demi-dose complémentaire d'insecticides dans le cas de la lutte raisonnée. Les applications insecticides à demi-dose sont ainsi réalisées avec une fréquence de 7 jours au cas où les seuils sont atteints en lutte raisonnée et une fréquence de 14 jours lorsque les seuils ne sont pas atteints. Compte tenu de la résistance de $H$. armigera aux pyréthrinoïdes, les insecticides ciblant ce ravageur ont été toujours appliqués à pleine dose en lutte raisonnée. Dans chaque parcelle élémentaire, la récolte du coton graine a été effectuée sur 40 lignes dont chaque ligne mesure 20 mètres de longeur.

Analyses statistiques : Les données collectées ont été statistiquement analysées avec le logiciel STAT-ITCF (ITCF, Boigneville, France). Le test de Newman-Keuls a été utilisé pour comparer les moyennes des paramètres 

culture cotonnière au Bénin

observés. Quand il y a une différence significative entre les moyennes des paramètres observés, les lettres $a, b$, et $c$ sont utilisées pour faire le classement; ainsi les

\section{RESULTATS}

Sur la station de recherche de Gobé dans le Centre Bénin, les paramètres observés et qui ont permis de discriminer les différentes doses de cyperméthrine et de paramètres possédant la même lettre ne sont pas significativement différents.

triazophos dans l'essai de réduction des doses de ces produits sont présentés dans les tableaux 1, 2 et 3 .

Tableau 1 : Pourcentages de capsules vertes saines, pourries, trouées et piquées durant l'essai d'étude de réduction de dose de cyperméthrine-triazophos.

\begin{tabular}{|c|c|c|c|c|c|c|c|c|}
\hline \multirow{2}{*}{$\begin{array}{l}\text { Doses des } \\
\text { produits } \\
\text { (g/ha) }\end{array}$} & \multicolumn{8}{|c|}{ Pourcentages de capsules vertes } \\
\hline & \multicolumn{2}{|c|}{ Saines } & \multicolumn{2}{|c|}{ Pourries } & \multicolumn{2}{|c|}{ Trouées } & \multicolumn{2}{|c|}{ Piquées } \\
\hline \begin{tabular}{l|l} 
Tri & Cyp
\end{tabular} & $7^{\text {ème }}$ obs & $\begin{array}{c}\text { Moyenne } \\
\text { (8 obs) }\end{array}$ & $7^{\text {ème }}$ obs & $\begin{array}{c}\text { Moyenne } \\
\text { (8 obs) }\end{array}$ & $6^{\text {ème }}$ obs & $\begin{array}{c}\text { Moyenne } \\
\text { (8 obs) }\end{array}$ & $7^{\text {ème }}$ obs & $\begin{array}{c}\text { Moyenne } \\
\text { (8 obs) }\end{array}$ \\
\hline$\overline{75}$ & 75,2 & 79,5 & $0,9 \mathrm{~b}$ & $0,6 \mathrm{~b}$ & $5,8 \mathrm{~b}$ & 9,6 & $11,4 \mathrm{~b}$ & 10,3 \\
\hline 150 & 77,4 & 81,0 & $0,5 \mathrm{a}$ & $0,5 \mathrm{a}$ & $3,6 a$ & 8,7 & $9,8 \mathrm{a}$ & 10,0 \\
\hline 0 & $65,3 \mathrm{c}$ & $74,2 \mathrm{c}$ & $1,8 \mathrm{~b}$ & $0,9 \mathrm{~b}$ & $14,0 \mathrm{~b}$ & $12,4 \mathrm{c}$ & $15,6 \mathrm{c}$ & $12,9 \mathrm{c}$ \\
\hline 10 & $69,5 b c$ & $77,5 \mathrm{bc}$ & $0,5 a$ & $0,6 a b$ & $15,3 \mathrm{~b}$ & $11,1 \mathrm{bc}$ & $12,8 \mathrm{bc}$ & $10,9 \mathrm{~b}$ \\
\hline 15 & $75,8 \mathrm{ab}$ & $80,5 a b$ & $0,7 \mathrm{a}$ & $0,6 a b$ & $9,5 \mathrm{a}$ & $9,0 a b$ & $10,3 a b$ & $10,0 a b$ \\
\hline 20 & $82,2 \mathrm{a}$ & $81,7 a b$ & $0,4 \mathrm{a}$ & $0,6 a b$ & $7,2 \mathrm{a}$ & $8,4 a b$ & $7,3 a$ & $9,3 a$ \\
\hline 30 & $82,8 \mathrm{a}$ & $83,7 \mathrm{a}$ & $0,3 a$ & $0,4 \mathrm{a}$ & $8,4 \mathrm{a}$ & $7,0 a$ & $7,7 \mathrm{a}$ & $8,9 a$ \\
\hline 35 & $82,1 \mathrm{a}$ & 83,7 a & $0,2 \mathrm{a}$ & $0,4 \mathrm{a}$ & $7,3 \mathrm{a}$ & $6,9 a$ & 10,1 ab & $9,0 \mathrm{a}$ \\
\hline 750 & 63,0 & 71,9 & 1,2 & 0,9 & 11,8 & 13,8 & 15,7 & 13,8 \\
\hline 7510 & 68,2 & 76,5 & 0,7 & 0,9 & 19,0 & 11,5 & 13,0 & 11,1 \\
\hline 7515 & 75,0 & 79,9 & 0,2 & 0,5 & 8,3 & 9,7 & 12,0 & 9,9 \\
\hline 7520 & 83,5 & 82,1 & 0,5 & 0,7 & 7,7 & 8,1 & 7,3 & 9,0 \\
\hline 7530 & 80,7 & 83,2 & 0,0 & 0,4 & 9,7 & 7,4 & 9,3 & 9,0 \\
\hline 7535 & 81,0 & 83,2 & 0,2 & 0,5 & 8,0 & 6,9 & 11,2 & 9,3 \\
\hline 1500 & 67,7 & 76,4 & 2,3 & 0,9 & 16,2 & 11,0 & 15,5 & 12,0 \\
\hline 15010 & 70,8 & 78,5 & 0,3 & 0,4 & 11,7 & 10,6 & 12,5 & 10,8 \\
\hline 15015 & 76,7 & 81,1 & 1,2 & 0,6 & 10,7 & 8,2 & 8,5 & 10,2 \\
\hline 15020 & 80,8 & 81,3 & 0,3 & 0,5 & 6,7 & 8,7 & 7,3 & 9,6 \\
\hline 15030 & 85,0 & 84,2 & 0,7 & 0,4 & 7,2 & 6,7 & 6,0 & 8,8 \\
\hline 15035 & 83,2 & 84,2 & 0,2 & 0,2 & 6,5 & 6,9 & 9,0 & 8,7 \\
\hline
\end{tabular}

Tri $=$ Triazophos $;$ Cyp $=$ Cyperméthrine $;$ Obs $=$ Observation

Les observations réalisées à des dates ponctuelles ne sont pas mentionnées si elles ne sont pas discriminantes. Par exemple la deuxième observation des capsules vertes saines n'est pas présentée. Les valeurs possédant la même lettre a, b ou c ne sont pas significativement différentes.

Le dénombrement des capsules saines, pourries, trouées et piquées a mis en évidence une différence significative $(p<0,001)$ entre les six doses de cyperméthrine testées et aucune différence significative n'a été observée entre les doses allant de $15 \mathrm{~g} / \mathrm{ha}$ à $35 \mathrm{~g} / \mathrm{ha}$ pour ce produit (tableau 1). Les capsules saines dénombrées n'ont pas permis de discriminer les deux doses de triazophos testées. Par contre, les capsules pourries, trouées par les chenilles et piquées par les punaises montrent que la dose de $75 \mathrm{~g} / \mathrm{ha}$ de triazophos est significativement inférieure $(p<0,05)$ à la dose de $150 \mathrm{~g} /$ ha (tableau 1). L'interaction entre les doses de cyperméthrine et de triazophos n'a pas été significative dans cet essai. Parmi les ravageurs dénombrés dans cet essai, seuls $C$. 

culture cotonnière au Bénin

leucotreta et $P$. gossypiella ont mis en évidence une

cyperméthrine

testées

(tableau

2).

différence significative $(p<0,005)$ entre les six doses de

Tableau 2 : Nombre de chenilles $C$. leucotreta et $P$. gossypiella dans 100 capsules vertes durant l'essai d'étude de réduction de dose de cyperméthrine-triazophos.

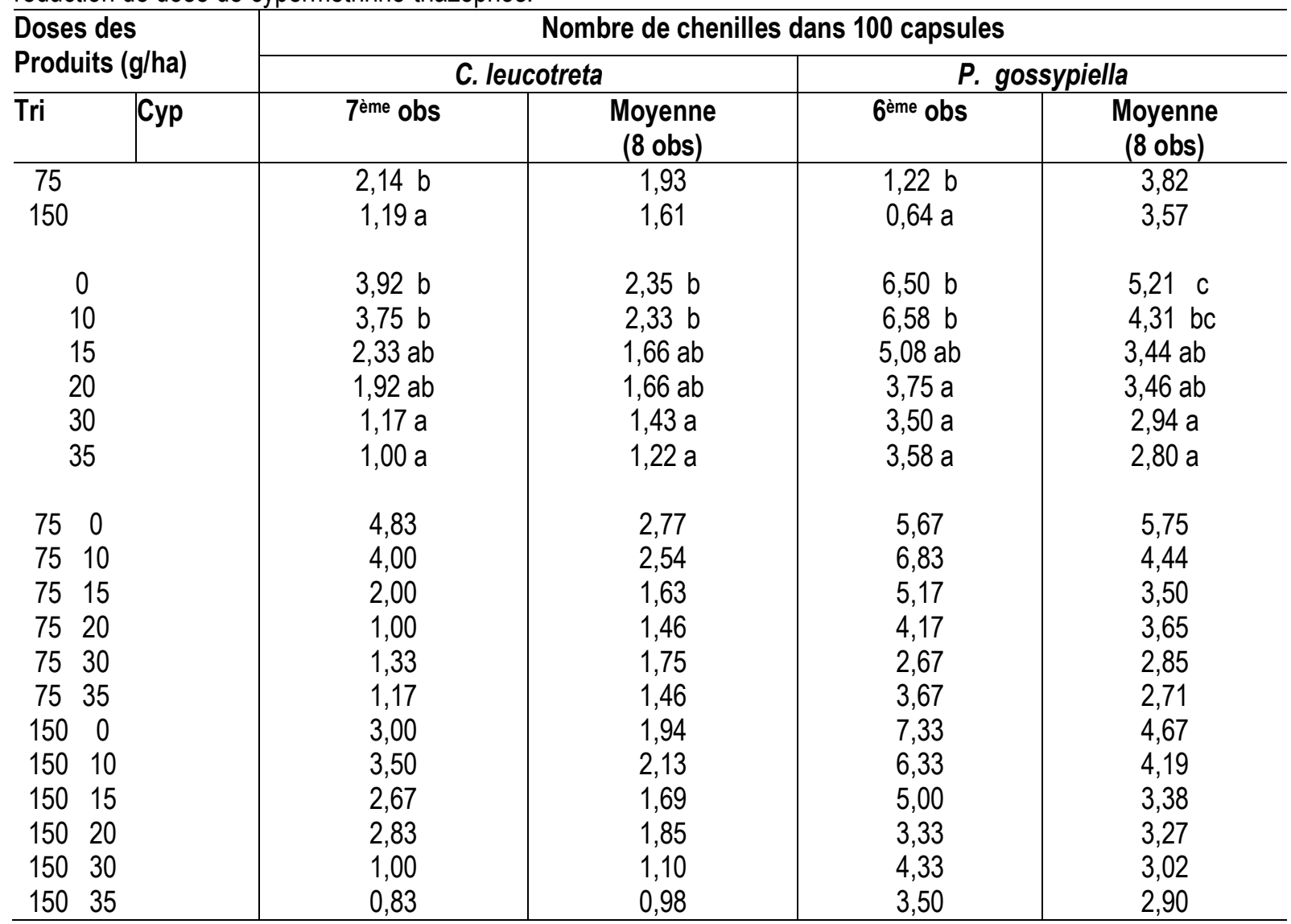

Tri = Triazophos $;$ Cyp $=$ Cyperméthrine $;$ Obs = Observation

Les observations réalisées à des dates ponctuelles ne sont pas mentionnées si elles ne sont pas discriminantes. Par exemple la quatrième observation des chenilles de $C$. leucotreta n'est pas présentée. Les valeurs possédant la même lettre a, b ou c ne sont pas significativement différentes.

Comme au niveau des capsules vertes dénombrées, aucune différence significative n'a été observée entre les doses de cyperméthrine allant de $15 \mathrm{~g} / \mathrm{ha}$ à $35 \mathrm{~g} / \mathrm{ha}$ lors des dénombrements des chenilles de $C$. leucotreta et de $P$. gossypiella dans les capsules vertes. Quant au triazophos, la dose de $75 \mathrm{~g} / \mathrm{ha}$ de ce produit est significativement inférieure $(p<0,05)$ à la dose de 150 $\mathrm{g} /$ ha durant le sixième dénombrement de $P$. gossypiella et le septième dénombrement de $C$. leucotreta dans les capsules vertes (tableau 2). A la première et à la deuxième récoltes du coton graine ainsi qu'à la récolte totale (tableau 3), quand bien même une différence significative a été observée entre les six doses de cyperméthrine expérimentées, aucune différence significative n'a été obtenue entre les rendements fournis par les doses $20 \mathrm{~g} / \mathrm{ha}, 30 \mathrm{~g} / \mathrm{ha}$ et $35 \mathrm{~g} / \mathrm{ha}$ de ce produit. Concernant le triazophos, aucune différence significative n'a été obtenue entre les rendements fournis par les deux doses $(75 \mathrm{~g} / \mathrm{ha}$ et $150 \mathrm{~g} / \mathrm{ha})$. En milieu réel, dans chacune des communes cotonnières de Dassa, N'Dali, Nikki et Banikoara, la lutte raisonnée basée sur l'application de demi-dose d'insecticides avec l'apport de la demi-dose complémentaire en fonction du seuil d'infestation des ravageurs a permis d'obtenir un rendement de coton graine significativement supérieur $(p<0,01)$ à celui de la protection classique (tableau 4). 

culture cotonnière au Bénin

Tableau 3 : Rendement de coton graine durant l'essai d'étude de réduction de dose de cyperméthrine-triazophos.

\begin{tabular}{|c|c|c|c|c|c|}
\hline \multicolumn{2}{|c|}{$\begin{array}{l}\text { Doses des } \\
\text { produits } \\
\text { (g/ha) }\end{array}$} & \multicolumn{4}{|c|}{ Rendement de coton-graine (kg/ha) } \\
\hline Tri & Сур & 1ère Récolte & 2 $^{\text {ème }}$ Récolte & $3^{\text {ème }}$ Récolte & Récolte totale \\
\hline $\begin{array}{l}75 \\
150\end{array}$ & & $\begin{array}{l}738 \\
800\end{array}$ & $\begin{array}{l}533 \\
579\end{array}$ & $\begin{array}{l}369 \\
371\end{array}$ & $\begin{array}{l}1640 \\
1750\end{array}$ \\
\hline 0 & & $621 \mathrm{~b}$ & $414 \mathrm{c}$ & 295 & $1330 \mathrm{c}$ \\
\hline 10 & & $688 a b$ & $433 \mathrm{c}$ & 355 & $1476 \mathrm{c}$ \\
\hline 15 & & $731 a b$ & 539 bc & 413 & $1683 b$ \\
\hline 20 & & $877 \mathrm{a}$ & $595 a b$ & 369 & $1841 \mathrm{ab}$ \\
\hline 30 & & $830 \mathrm{a}$ & $629 a b$ & 399 & $1858 \mathrm{ab}$ \\
\hline 35 & & 869 a & $728 \mathrm{a}$ & 390 & 1987 a \\
\hline 750 & & 602 & 432 & 282 & 1316 \\
\hline 7510 & & 657 & 392 & 326 & 1375 \\
\hline 7515 & & 674 & 561 & 388 & 1623 \\
\hline 7520 & & 843 & 591 & 337 & 1771 \\
\hline 7530 & & 811 & 578 & 456 & 1845 \\
\hline 7535 & & 843 & 646 & 424 & 1913 \\
\hline 1500 & & 640 & 396 & 307 & 1343 \\
\hline 15010 & & 718 & 473 & 384 & 1575 \\
\hline 15015 & & 788 & 517 & 438 & 1743 \\
\hline 15020 & & 911 & 598 & 402 & 1911 \\
\hline 15030 & & 848 & 680 & 341 & 1869 \\
\hline 15035 & & 896 & 811 & 356 & 2063 \\
\hline
\end{tabular}

Tri = Triazophos $;$ Cyp = Cyperméthrine

Trois récoltes échelonnées ont été effectuées. Les valeurs possédant la même lettre $a, b$ ou c ne sont pas significativement différentes.

Tableau 4 : Rendements de coton graine en protection classique et en lutte raisonnée en milieu réel dans quatre communes des bassins cotonniers du Bénin : Dassa, N'Dali, Nikki et Banikoara. Les valeurs possédant la même lettre a ou b ne sont pas significativement différentes.

\begin{tabular}{l|c|c|c|c}
\hline \multirow{2}{*}{ Stratégies de protection } & \multicolumn{4}{|c}{ Rendements de coton graine (kg/ha) } \\
\cline { 2 - 5 } & Dassa* & N'Dali & Nikki & Banikoara \\
\hline \multirow{2}{*}{$\begin{array}{l}\text { Protection classique } \\
\text { Lutte raisonnée }\end{array}$} & $1165 \mathrm{~b}$ & $1264 \mathrm{~b}$ & $1394 \mathrm{~b}$ & $1933 \mathrm{~b}$ \\
\hline
\end{tabular}

* Dans la commune de Dassa, la cyperméthrine a été utilisée à pleine dose en lutte raisonnée comme en lutte classique.

\section{DISCUSSION}

La pression de sélection insecticide est un facteur important du processus d'apparition de la résistance des insectes aux insecticides. La pleine dose de cyperméthrine appliquée durant plusieurs années en Afrique de l'Ouest et du Centre en général et au Bénin en particulier a entraîné l'apparition de la résistance de $H$. armigera aux pyréthrinoïdes dans ces régions (Martin et al., 2005 ; Djihinto et al., 2009 et 2012 ; Achaleke et Brévault, 2010). La lutte raisonnée expérimentée en culture cotonnière au Bénin à l'instar de la lutte étagée ciblée, la lutte sur seuil, la lutte après observation individuelle des chenilles appliquées en Afrique de 
l'Ouest et du Centre visant à réduire la dose d'insecticide épandu (Silvie et Sognigbe, 1993; Vodounnon, 1995; Nibouche et al., 2003; Sinzogan et al., 2004; Prudent et al., 2007; Silvie et al., 2011 et 2013 ; Kpadé et Mensah, 2013; Brévault et al., 2014 ) est une stratégie de réduction de la pression de sélection insecticide et un outil de prévention et de gestion de la résistance des insectes aux insecticides (Djihinto, 2004; Djihinto et al., 2009 et 2013). Au Bénin, un faible niveau de résistance de $H$. armigera aux pyréthrinoïdes a été observé dans les zones où la stratégie de lutte raisonnée avec la réduction de la pression de sélection insecticide a été adoptée et les niveaux de résistance à la cyperméthrine du ravageur diminuent pendant les périodes de réduction des traitements insecticides (Djihinto et al., 2009). L'application des produits insecticides ayant souvent fait l'objet d'intoxication des agriculteurs (Tovignan et al., 2001; Prudent et al., 2007) et des insectes auxiliaires non ciblés (Colignon et al., 2003), l'utilisation de demi-dose d'insecticides en lutte raisonnée diminue la quantité de produit épandu quand les seuils ne sont pas atteints et peut contribuer à la réduction des cas d'intoxication des populations humaines et la diminution de la pression insecticide exercée sur la faune utile. Sur le plan économique, le coût des nouvelles matières actives insecticides sont relativement élevés et un autre avantage de la lutte raisonnée visant à réduire la dose des insecticides appliqués réside dans la diminution des coûts de protection par la réduction de la quantité de produits utilisés lorsque les seuils des ravageurs ne sont pas atteints. En culture cotonnière au Bénin, la cyperméthrine est appliquée à pleine dose à $35 \mathrm{~g} / \mathrm{ha}$ (sans association avec d'autres insecticides) et à $30 \mathrm{~g} / \mathrm{ha}$ lorsqu'elle est associée à un organophosphoré. L'essai de réduction de dose a montré que les doses de cyperméthrine allant de 15 à $35 \mathrm{~g} / \mathrm{ha}$ ne sont pas significativement différentes pour le contrôle des infestations des ravageurs et la production des capsules vertes saines. De même, les doses de cyperméthrine

\section{CONCLUSION}

L'essai de réduction de dose sur station de recherche montre que la demi-dose de cyperméthrine suffit pour maîtriser les infestations des ravageurs notamment $C$. leucotreta et $P$. gossypiella. Cependant, l'application de la demi-dose de cyperméthrine ne garantit pas toujours un rendement satisfaisant de coton graine et l'épandage d'une demi-dose complémentaire peut être nécessaire en fonction du niveau d'infestation des ravageurs. Quant au triazophos, l'application d'une demi-dose de ce produit n'a pas permis de contrôler les infestations des ravageurs allant de 20 à $35 \mathrm{~g} / \mathrm{ha}$ ne sont pas significativement différentes lorsqu'on considère les rendements de coton graine obtenus. Ces résultats montrent que la réduction de la pleine dose de cyperméthrine $35 \mathrm{~g} / \mathrm{ha}$ est possible et ne contredisent pas la possibilité de réduire de moitié la dose d'insecticide signalée par Amadou et Sidi (2013) dans le domaine de la lutte contre les invasions acridiennes, tout en conservant la même efficacité des produits appliqués. La pleine dose de triazophos appliquée dans la grande zone de production cotonnière au Bénin est $150 \mathrm{~g} / \mathrm{ha}$. Des différences significatives ont été parfois mises en évidence entre les deux doses de triazophos testées (75 et $150 \mathrm{~g} / \mathrm{ha}$ ). La réduction de moitié de la dose de triazophos ne peut donc être effectuée sans envisager l'apport de la demi-dose complémentaire. Quant à la cyperméthrine, la réduction de la pleine dose $35 \mathrm{~g} / \mathrm{ha}$ à $20 \mathrm{~g} / \mathrm{h}$ a peut être effectuée sans craindre une chute significative de rendement de coton graine alors que l'application de la demi-dose de ce produit ne peut se faire sans envisager l'apport d'une dose complémentaire. En milieu réel, l'application de demi-dose d'insecticides avec l'apport de la demi-dose complémentaire en lutte raisonnée a permis d'améliorer significativement les rendements en coton graine. Cette amélioration des rendements en lutte raisonnée au Bénin a été également observée dans plusieurs pays comme le Cameroun, le Tchad, la Côte d'Ivoire, le Burkina Faso (Vodounnon, 1995 ; Nibouche et al., 2003 ; Silvie et al., 2011 et 2013). Cet accroissement des rendements peut être dû à une attention particulière accordée par les producteurs aux parcelles cotonnières en lutte raisonnée lors des opérations d'entretien de la culture (Silvie et al., 2013). L'augmentation de la fréquence des applications insecticides en lutte raisonnée par l'utilisation hebdomadaire de demi-dose peut aussi expliquer cet accroissement de rendement de coton graine par rapport à l'application de la pleine dose avec une fréquence de 14 jours en protection classique.

rencontrés. La demi-dose complémentaire du triazophos peut être nécessaire en fonction du seuil d'infestation des ravageurs pour une production satisfaisante du coton. En milieu réel, la lutte raisonnée basée sur l'application de demi-dose d'insecticides avec l'apport de la demi-dose complémentaire en fonction du seuil d'infestation des ravageurs a permis d'obtenir un rendement de coton graine significativement supérieur $(p<0,01)$ à celui de la protection classique. Cette stratégie de lutte raisonnée en culture cotonnière mérite d'être encouragée. 


\section{REMERCIEMENTS}

Nous remercions le Directeur Mensah Guy Apollinaire pour la lecture du manuscrit et les commentaires effectués. Nous remercions également les firmes Bayer

\section{REFERENCES}

Achaleke J et Brévault T, 2010. Inheritance and stability of pyrethroid resistance in the cotton bollworm Helicoverpa armigera (Lepidoptera : Noctuidae) in Central Africa. Pest Manag. Sci. 66: 137-141.

Amadou BB et Sidi MS, 2013. Réduction des doses efficaces d'insecticides contre les larves de criquet pèlerin (Schistocerca gregaria Forskal, 1775 : Orthoptera, Acrididae) par utilisation de quantités réduites de phénylacétonitrile, Base [En ligne] 17: 572-579. URL: http://popups.ulg.ac.be/17804507/index. php?id=10367.

Brévault $T$, Renou A, Vayssières J-F, Amadji G, Assogba-Komlan F, Diallo MD, De Bon H, Diarra K, Hamadoun A, Huat J, Marnotte P, Menozzi P, Prudent P, Rey J-Y, Sall D, Silvie P., Simon S, Sinzogan A, Soti V, Tamo M, Clouvel P, 2014. Divecosys: Bringing together researchers to design ecologically-based pest management for small-scale farming systems in West Africa. Crop Protection 66: 53-60.

Colignon P, Haubruge E, Gaspar C, Francis F, 2003. Effets de la réduction de doses de formulations d'insecticides et de fongicides sur l'insecte auxiliaire non ciblé Episyrphus balteatus (Diptera : Syrphidae). Phytoprotection 84: 141148.

Djihinto CA, 2004. La résistance de $H$. armigera aux pyréthrinoïdes en culture cotonnière au Bénin : du mécanisme, du coût biologique et des stratégies de gestion de la résistance. Thèse de doctorat unique, Université de Cocody Abidjan Côte d'lvoire, 259 pp.

Djihinto CA, Hougni A, Houngbo EN, Katary A, BokononGanta HA, 2013. Insecticide resistance fitness cost and resistance stability. International Journal of Tropical Agriculture and Food Systems $7: 17-29$.

Djihinto CA, Katary A, Djaboutou CM, Prudent $\mathrm{P}$, Menozzi P, Atachi P, 2012. Variation in biological parameters of cypermethrin resistant and susceptible strains of Helicoverpa armigera from Benin Republic. West Africa. Int. J. Biol. Chem. Sci. 6 : 931-940. et Arysta Life Science pour avoir fourni les insecticides expérimentés et pour leur franche collaboration.

Djihinto CA, Katary A, Prudent P, Vassal J-M, Vaissayre $M, 2009$. Variation in resistance to pyrethroids in Helicoverpa armigera from Benin Republic, West Africa. Journal of Economic Entomology $102: 1928-1934$.

Kpadé PC et Mensah ER, 2013. Facteurs d'adoption de la lutte étagée ciblée au Nord-Bénin. Économie Rurale $338: 77-91$.

Martin T, Ochou OG, Djihinto CA, Traore D, Togola M, Vassal J-M, Vaissayre M, Fournier D, 2005. Controlling an insecticide-resistant bollworm in West Africa. Agriculture, Ecosystems and Environment 107 : 409-411.

Nibouche S, Beyo J, Djonnewa A, Goipaye I, Yandia A, 2003. La lutte étagée ciblée a-t-elle un avenir en Afrique centrale ? In : Jamin JY, Seiny Boukar L, Floret C, (Eds.), Savanes africaines: des espaces en mutation, des acteurs face à de nouveaux défis. Actes du colloque, Garoua, Cameroun, 27-31 mai 2002.CIRAD, Montpellier, $9 \mathrm{pp}$.

Prudent P, Loko S, Deybe D, Vaissayre M, 2007. Factors limiting the adoption of IPM practices by cotton farmers in Bénin : a participatory approach. Expl Agric. $\quad 43: \quad 113-124 . \quad$ Doi : 10.1017/S00144797006004261

Silvie PJ, Adegnika MA, Akantetou KP, Ayeva B, Bonni G, Brevault T, Gautier C, Héma O, Houndete TA, Ochou G, Prudent P, Renou A, Togola M, 2011. Cotton pest management programmes using threshold-based interventions developed by CIRAD and its partners in sub-saharan African countries. World cotton research conference on technologies for prosperity, pp. 244-253.

Silvie PJ, Renou A, Vodounnon S, Bonni G, Adegnika MO, Héma $O$, Prudent $P$, Sorèze J, Ochou OG, Togola M, Badiane D, Ndour A, Akantetou PK, Ayeva B, Brévault T, 2013. Threshold-based interventions for cotton pest control in West Africa: what's up 10 years later? Crop Protection 43: 157-165.

Silvie PJ et Sognigbe B, 1993. Use of action thresholds on cotton crops in northern Togo. International Journal of Pest Management $39:$ 51-56. 
Djihinto et al. J. Appl. Biosci. 2016 Réduction de doses de cyperméthrine- triazophos et lutte raisonnée en culture cotonnière au Bénin

Sinzogan AAC, Huis Van A, Kossou DK, Jiggins J, Vodouhè S, 2004. Farmers' knowledge and perception of cotton pests and pest control practices in Benin : results of a diagnostic study. NJAS 52: 285-303.

Tovignan S, Vodouhè S, Dinham B, 2001. Cotton pesticides cause more deaths in Benin. Pesticides News 52: 12-14.

Vodounnon S, 1995. La lutte étagée ciblée en culture cotonnière au Bénin. Bulletin de la Recherche Agronomique 12 : 6-14. 